\title{
How well do force fields capture the strength of salt bridges in proteins?
}

\author{
Mustapha Carab Ahmed ${ }^{1}$, Elena Papaleo ${ }^{1,2}$, Kresten Lindorff-Larsen ${ }^{\text {Corresp. } 1}$ \\ ${ }^{1}$ Structural Biology and NMR Laboratory, Linderstrøm-Lang Centre for Protein Science, Department of Biology, University of Copenhagen, Copenhagen, \\ Denmark \\ 2 Computational Biology Laboratory, Danish Cancer Society Research Center, Copenhagen, Denmark \\ Corresponding Author: Kresten Lindorff-Larsen \\ Email address: lindorff@bio.ku.dk
}

Salt bridges form between pairs of ionisable residues in close proximity and are important interactions in proteins. While salt bridges are known to be important both for protein stability, recognition and regulation, we still do not have fully accurate predictive models to assess the energetic contributions of salt bridges. Molecular dynamics simulation is one technique that may be used study the complex relationship between structure, solvation and energetics of salt bridges, but the accuracy of such simulations depends on the force field used. We have used NMR data on the B1 domain of protein G (GB1) to benchmark molecular dynamics simulations. Using enhanced sampling simulations, we calculated the free energy of forming a salt bridge for three possible lysine-carboxylate ionic interactions in GB1. The NMR experiments showed that these interactions are either not formed, or only very weakly formed, in solution. In contrast, we show that the stability of the salt bridges is overestimated, to different extents, in simulations of GB1 using seven out of eight commonly used combinations of fixed charge force fields and water models. We also find that the Amber ff15ipq force field gives rise to weaker salt bridges in good agreement with the NMR experiments. We conclude that many force fields appear to overstabilize these ionic interactions, and that further work may be needed to refine our ability to model quantitatively the stability of salt bridges through simulations. We also suggest that comparisons between NMR experiments and simulations will play a crucial role in furthering our understanding of this important interaction. 
1 How well do force fields capture the strength of salt bridges in proteins?

3

4

\section{Corresponding Author}

11 Kresten Lindorff-Larsen

12 Email address: lindorff@bio.ku.dk

13

\section{Present Address}

$15{ }^{2}$ Computational Biology Laboratory, Danish Cancer Society Society Research Center, Copenhagen, Denmark 


\section{Abstract}

Salt bridges form between pairs of ionisable residues in close proximity and are important interactions in proteins. While salt bridges are known to be important both for protein stability, recognition and regulation, we still do not have fully accurate predictive models to assess the energetic contributions of salt bridges. Molecular dynamics simulation is one technique that may be used study the complex relationship between structure, solvation and energetics of salt bridges, but the accuracy of such simulations depends on the force field used. We have used NMR data on the B1 domain of protein $\mathrm{G}(\mathrm{GB} 1)$ to benchmark molecular dynamics simulations. Using enhanced sampling simulations, we calculated the free energy of forming a salt bridge for three possible lysine-carboxylate ionic interactions in GB1. The NMR experiments showed that these interactions are either not formed, or only very weakly formed, in solution. In contrast, we show that the stability of the salt bridges is overestimated, to different extents, in simulations of GB1 using seven out of eight commonly used combinations of fixed charge force fields and water models. We also find that the Amber ff15ipq force field gives rise to weaker salt bridges in good agreement with the NMR experiments. We conclude that many force fields appear to overstabilize these ionic interactions, and that further work may be needed to refine our ability to model quantitatively the stability of salt bridges through simulations. We also suggest that comparisons between NMR experiments and simulations will play a crucial role in furthering our understanding of this important interaction.

\section{Introduction}

Proteins are stabilized via the concerted action of numerous weak forces including those that arise from hydrogen bonds, the hydrophobic effect and salt bridges (Dill, 1990; Zhou \& Pang, 2017). While we now know much about the relative contributions and physical origins of these effects, we still do not have quantitative models that allow us, for example, to predict accurately the overall stability of a protein given its three-dimensional structure. A quantitative understanding of protein stability would aid both to design proteins with improved stabilities (Foit et al., 2009), as well as understand how loss of protein stability may give rise to disease (Casadio et al., 2011; Nielsen et al., 2017).

We here focus our attention on salt bridges in proteins, where they occur between oppositely charged ionisable groups, most commonly between the negatively charged side chains of aspartate and glutamate or the carboxy-terminus, and positive charges in arginine, lysine or histidine side chains, or with the N-terminal ammonium group. A salt bridge is formed when the two residues involved are spatially close enough to form energetically favourable electrostatic interaction between the (partially) charged atoms (Barlow \& Thornton, 1983). Although the first quantitative models of protein electrostatics are almost one hundred years old (LinderstrømLang, 1924), our ability to predict electrostatic properties remain incomplete. Indeed, while ionic interactions were also early suggested to contribute substantially to the stability of certain proteins (Speakman, J. B., \& Hirst, 1931), their importance and energetic contribution was controversial already before they had been observed in experimentally-derived protein structures (Jacobsen \& Linderstrøm-Lang, 1949).

The stability of a salt bridge depends on the environment around the residues, the $\mathrm{pH}$, and the distance and geometric orientation between the involved residues. Salt bridges may be located on the protein surface, where they are exposed to the solvent, or they can be found buried within the hydrophobic interior of the folded protein. Studies on different protein families have found that 
61 buried salt-bridges are more likely to be conserved and functionally relevant than surface

62 exposed ones (Schueler \& Margalit, 1995; Takano et al., 2000). Surface exposed salt bridges are

63 generally weaker, more variable in their contribution to stability, and more difficult to predict

64 (Sarakatsannis \& Duan, 2005). Double mutant cycles in barnase illustrate the distinction between

65 forming a salt bridge in the low dielectric environment of the protein core versus the higher

66 dielectric on the protein surface. These experiments show that an internal salt bridge stabilizes

67 the protein by more than $3 \mathrm{kcal} \mathrm{mol}^{-1}$ (Vaughan et al., 2002), whereas a surface exposed salt

68 bridge is about 10 times weaker, $\sim 0.3 \mathrm{kcal} \mathrm{mol}^{-1}$ at low ionic strength, a value that decreases to

69 zero at higher ionic strength (Serrano et al., 1990).

70 thus not always clear whether these interactions are stable in solution, in particular because their dynamic and transient nature make them difficult to study. With its site-specific resolution and ability to detect even transient interactions, NMR spectroscopy can, however, be used to study salt bridges in solution, and to determine the extent to which different residues interact. In one intriguing study, a range of different NMR experiments were used to examine three different potential salt bridges in the B1 domain of Protein G (hereafter called GB1) (Tomlinson et al., 2009). Six different crystal structures of GB1 show that three of six solvent-exposed lysines, K12, K39 and K58, form salt-bridges to the nearby acidic residues E12, E23 and D55 (Tomlinson et al., 2009) (Fig. 1A). To examine whether these ionic interactions are present in solution, the authors performed a series of experiments that include monitoring both the lysine nitrogen and proton chemical shifts, and the hydrogen-deuterium isotope effects on the ammonium group, while titrating the carboxylates to protonate them. Surprisingly, for two of the putative ion pairs (K12-E23 and K58-D55) the results showed essentially no observable changes at the lysines as the carboxylates changed protonation state, suggesting no substantial formation of a salt bridge. For one pair, the intra-helical K39-E35, there was a small change in the ${ }^{15} \mathrm{~N}$ chemical shift of the ammonium group as $\mathrm{pH}$ was varied to protonate $\mathrm{E} 35$ (and other carboxylates), though no change was observed for the ${ }^{1} \mathrm{H}$ chemical shift nor for the isotope effects. These observations were also supported by the determination of the pKa values of the carboxylate and ammonium groups in these residues. The authors therefore conclude that two of the salt bridges (K12-E23 and K58-D55) are not formed in solution, while the third (K39-E35) may be weakly formed (though likely at a low population). Computational methods provide an alternative approach to study the structure and dynamics of ionic interactions in solution (Kumar \& Nussinov, 2002). All-atom, explicit solvent molecular dynamics (MD) simulations, in particular, can be used to provide insight into the structure and energetics of biomolecules in an aqueous environment. For such simulations to provide an accurate description of salt bridges it is, however, important that the energy function (force field) used provides an accurate description of the balance between the many forces that determine salt bridge strengths. As part of the re-parameterization and validation of one of the more accurate force fields, CHARMM22*, we discovered that ionic interaction between guanidinium and acetate ions were $\sim 10$ times too strong in the original CHARMM22 force field (Piana, LindorffLarsen \& Shaw, 2011). This observation led to a re-parameterization (the "DER correction") of the partial charges in Asp, Glu and Arg side chains, to bring the interactions closer to experiment (but still slightly overestimated) (Piana, Lindorff-Larsen \& Shaw, 2011). Building upon these ideas, Debiec and colleagues examined the interactions between analogues of both arginine, lysine and histidine side chains with carboxylates in a range of force field and water models (Debiec, Gronenborn \& Chong, 2014). Although they found considerable variability between 
107 different force fields, the general observation from the CHARMM force fields hold true, namely

108 that the ionic interactions between side chain analogues are overstabilized relative to experiment.

109 Such overstabilization is not without consequence when studying protein dynamics using

110 simulations. For example, in a study of voltage gating in potassium channels the simulations

111 were performed with the DER correction, or the even more substantial DER2 correction, in order

112 to be able to observe the switch in ionic interactions that are central in channel gating (Jensen et

113 al., 2012). Similarly, when using free energy perturbation MD simulations to predict the change

114 in protein stability from a mutation, the results are less accurate when the mutations involve a

115 change in charge (Steinbrecher et al., 2017).

116 In addition to the DER correction described above, several groups have examined and modified

117 the charges in protein force fields in order to provide a more accurate description of protein

118 electrostatics. For example, Jensen updated the parameters for the charged amino acids in the

119 OPLS force field to obtain a self-consistent set of parameters that reproduce experimental

120 hydration free energies for side chain analogues (Jensen, 2008). Debiec et al used the Implicitly

121 Polarized Charge (IPolQ) method to derive a new set of partial charges for Amber, resulting in

122 the ff15ipq force field, and showed that these modifications substantially improved the

123 agreement with experiments on the association between analogues of charged side chains

124 (Debiec et al., 2016).

125 Here we build on the work described above by examining the extent to which seven different

126 fixed charge force fields capture the behaviour of the three putative salt bridges in GB1. In

127 particular, we conducted explicit-solvent molecular simulations of GB1 using the Amber

128 ff99SB*-ILDN, Amber ff03w, CHARMM27, CHARMM22*, OPLS_2005, AMBER ff15ipq

129 and a99SB-disp force fields. In contrast to studies of side chain analogues, simulations of the salt

130 bridges in the context of a folded protein takes into account the natural geometrical and energetic

131 constraints imposed by the protein scaffold. To accelerate sampling and ensure convergence we

132 used metadynamics simulations to map the free energy surface of salt bridge formation. The

133 results reveal that also in the context of a folded protein most of these force fields overestimate

134 the population of ionic interactions, and also provide insight into the geometry of salt bridges in

135 proteins.

\section{Materials and Methods}

137 In order to investigate potential salt-bridging interactions between residues K12-E23, K39-E35

138 and K58-D55 in GB1 we performed both standard MD simulations as well as enhanced sampling

139 simulations using well-tempered metadynamics (WT-MetaD) (Barducci, Bussi \& Parrinello,

140 2008). To test the effect of the force field we used seven different force fields: Amber ff99SB*-

141 ILDN (Hornak et al., 2006; Best \& Hummer, 2009; Lindorff-Larsen et al., 2010), Amber ff03w

142 (Best \& Mittal, 2010), CHARMM27 (CHARMM22 with the CMAP correction) (MacKerell et

143 al., 1998; Mackerell, Feig \& Brooks, 2004), CHARMM22* (Piana, Lindorff-Larsen \& Shaw,

144 2011), OPLS 2005 (Banks et al., 2005), a99SB-disp (Robustelli, Piana \& Shaw, 2018) and

145 AMBER ff15ipq (Debiec et al., 2016) (Table 1). Each force field was combined with its "native"

146 water model, namely the CHARMM-specific TIP3P water model for CHARMM force fields

147 (MacKerell et al., 1998) (herein called TIPS3P), the original TIP3P water model (Jorgensen et

148 al., 1983) for the Amber ff99SB*-ILDN force fields, TIP4P/2005 (Abascal \& Vega, 2005) for

149 Amber ff03w and the SPC/E water model (Berendsen, Grigera \& Straatsma, 1987) for OPLS. In

150 the case of the AMBER ff15ipq force field, we used the SPC/ $\mathrm{E}_{\mathrm{b}}$ water model, and the simulation

151 box was constructed with AmberTools 17 (Case et al., 2017) and then converted to Gromacs 
152 format. A slightly modified version of the TIP4P-D water model (Piana et al., 2015) was used for

153 the a99SB-disp force field (Robustelli, Piana \& Shaw, 2018). We also examined the effect of the

154 water model by testing CHARMM22* with standard TIP3P.

\section{Setup and Equilibration}

156 The simulations with the updated AMBER ff15ipq and the a99SB-disp force fields were

157 performed with Gromacs version 2016.1 (Abraham et al., 2015), while the rest of simulations

158 were performed with Gromacs version 4.5 (van der Spoel et al., 2010), in both cases using the

159 crystal structure of GB1 (PDB ID 1PGB; (Gallagher et al., 1994) as starting structure. The

160 protein was centred and solvated in a dodecahedral box with an edge length of $12 \AA$, and the

161 resulting system consisted of the protein (56 residues), 5300 water molecules and 4 sodium ions

162 to neutralize the total charge. The cutoff for the Van der Waals interactions was $9 \AA$, while the

163 long-range electrostatic interactions were calculated using the particle mesh Ewald method with

164 a real-space cutoff of $12 \AA$. For each force field and water model, we first subjected the system to

$1650.2 \mathrm{~ns}$ energy minimization, followed by a $1 \mathrm{~ns}$ solvent equilibration with position restraints on

166 the protein backbone, followed by 1 ns of protein equilibration. After equilibration, we performed

167 a 10 ns simulation in the NPT ensemble (Parrinello \& Rahman, 1981, 1982; Bussi, Donadio \&

168 Parrinello, 2007) at $298 \mathrm{~K}$ and 1 atm pressure and calculated the average volume of the system.

169 Finally, we selected the frame with a volume closest to this average from the second half of these

$17010 \mathrm{~ns}$, and used this as starting point for WT-MetaD simulations in the NVT ensemble (Bussi,

171 Donadio \& Parrinello, 2007). The lengths of all bonds to hydrogen atoms were kept fixed using

172 the LINCS algorithm (Hess et al., 1997), and simulations were performed using a 2 fs time step.

\section{Molecular Dynamics Simulations}

174 Following equilibration, we performed a standard $100 \mathrm{~ns}$ unbiased MD simulation with each

175 force field. This was followed by three 100ns WT-MetaD simulations at 298K using the

176 PLUMED 1.3 plugin (Bonomi et al., 2009) with Gromacs 4.5 and PLUMED 2.3 plugin (Tribello

177 et al., 2014) with Gromacs 2016.1, and in each simulation enhancing separately the sampling of

178 one the three salt-bridges (K12-E23, K39-E35 and K58-D55). In metadynamics simulations,

179 sampling is enhanced by adding a history-dependent biasing potential along one or more selected

180 degree of freedom (collective variables, CVs). In order to sample the free energy of forming the

181 salt bridges, we used two CVs for each of the three ion-pairs (in three different simulations). For

182 the lysine-glutamate ion pairs we used the distance between the NZ-OE1 and NZ-OE2 atoms as

183 CVs, and for the lysine-aspartate pair we used the distance between the NZ-OD1 and NZ-OD2

184 atoms. For obvious symmetry reasons, the free energy surface should be identical for the two

185 NZ-oxygen pairs (e.g. NZ-OD1/OD2), and so the use of two CVs for each salt bridge allows us

186 both to map the interactions with various combinations of distances to the two oxygens, but also

187 to assess convergence via the similarity between the symmetry-related atoms. In the

188 metadynamics simulations, the biasfactor, sigma parameters, initial height of the Gaussian hills

189 and the deposition rate were set to $4,0.05 \AA, 0.12 \mathrm{kcal} \mathrm{mol}^{-1}$ and $2 \mathrm{ps,} \mathrm{respectively.} \mathrm{In} \mathrm{order} \mathrm{not}$

190 to enhance sampling of unrealistically long distances and to cause unfolding of GB1, we

191 introduced a restraining potential (a soft "wall") on the CVs, using the form $E_{\text {restr }}=k\left(d_{\mathrm{i}}-d_{\mathrm{max}}\right)^{4}$.

192 This potential acted whenever the distance, $d_{\mathrm{i}}$, was above $d_{\max }$, the longest distance observed in

193 the unbiased simulations (Table 1) and used a force constant of $k=4.8 \mathrm{kcal} \mathrm{mol}^{-1} \AA^{-4}$. Finally, to

194 test the robustness of the calculations, we also performed a 50-ns parallel-tempering

195 metadynamics (PT-MetaD) (Hansmann, 1997; Bussi et al., 2006) simulation for the K39-E35 
196 salt bridge using the CHARMM22* force field and TIPS3P water model. In particular, in the PT-

197 MetaD simulations, we further enhanced the sampling with exchanges between different

198 temperatures with a replica-exchange scheme. We used six replicas (at 294K, 298K, 308K,

$199322 \mathrm{~K}, 337 \mathrm{~K}$ and $353 \mathrm{~K}$ ) where the width of the energy distribution (of all but the "neutral" $298 \mathrm{~K}$

200 replica) was increased in a preparatory step of $10 \mathrm{~ns}$ as previously described (Sutto \& Gervasio,

201 2013). All replicas were also subjected to an additional biasing force through metadynamics with

202 the same parameters (initial height of the Gaussian hills, deposition rate and biasfactor) used in

203 the WT-MetaD simulation as described above.

204

205

206

207

208

209

210

211

\section{Analyses}

We analysed the salt bridge formation by creating two-dimensional free energy profiles for each salt bridge and for each force field, and using the two nitrogen-oxygen distances as coordinates. To quantify the formation of each salt bridge, we calculated the fraction of frames (after removing the metadynamics bias) where one of the two distances were below $5 \AA$.

\section{2 \\ 212 Results}

213 Molecular dynamics simulations of salt bridge formation

214 We performed MD simulations of GB1 using eight different combinations of force fields and

215

216

217

218

219

220

221

222

223

224

225

226

227 water models (Table 1) to examine the formation of salt bridges, and to benchmark against NMR experiments. While unbiased MD simulations showed reversible formation and breaking of the ionic interactions (Fig. 2A), we decided to use enhanced sampling metadynamics simulations (Fig. 2B) to ensure better convergence of the free energy of salt bridge formation. In both types of simulations, we find that the carboxylate and ammonium group spend time at multiple, relatively distinct sets of distances, though with differences between force fields and salt bridges (see below). Thus, for each of the eight different force field combinations we performed three metadynamics simulations, one for each salt bridge pair. In each of these 24 simulations, we simultaneously biased and monitored two CVs, corresponding to the distance between the ammonium nitrogen atom in the lysine side chain, and each of the two oxygen atoms in the carboxylate group in aspartate or glutamate. The metadynamics bias did not have any major effect on the protein stability over the course of the 100ns simulations. For example, the average backbone RMSD was below $1.5 \AA$ for both the unbiased and biased simulations (Fig. S1).

Free-energy surfaces and geometry of ionic interactions

We used the metadynamics simulations to reconstruct the free energy surface for salt bridge

230

231

232

233

234

235

236

237 formation along the two CVs (Fig. 1 and Fig. S2). The resulting profiles show a number of notable features. First, we find that they are highly symmetric across the "diagonal", as expected because of the equivalence of the two carboxylate oxygen atoms. Thus, while this behaviour is expected based on physical grounds, it provides a useful and independent test for convergence. Secondly, the profiles reveal a number of distinct free energy minima with depths of $\sim 1 \mathrm{kcal}$ $\mathrm{mol}^{-1}$ and separated by small free energy barriers. For example, in the case of the K12-E23 interaction in simulations using CHARMM22* and the TIPS3P water model (Fig. 1B), three minima are visible at short distances between the two charged side chains. Two of these are

238 symmetrically placed on either side of the diagonal and correspond to one oxygen-nitrogen 
239 distance around $3.0 \AA$ and the other at $\sim 4.5 \AA$. These minima thus correspond to a salt bridge

240 where one oxygen is close to the nitrogen (with a proton from the ammonium group in between)

241 and the other oxygen further away. A third minimum is also observed where both oxygen-

242 nitrogen distances are short $(\sim 2.5 \AA-3.0 \AA)$, corresponding to a different salt bridge geometry.

243 These three minima correspond to so called contact ion pairs where the cation and anion are in

244 direct contact. In some force fields, in particular for the K39-E35 and K58-D55 pairs, additional

245 minima are observed where the shortest of the two oxygen-nitrogen distances is $\sim 6 \AA-10 \AA$

246 (Figs. 1C, 1D and S2). These correspond to solvent-separated ion pairs, where one or more

247 solvent molecules sit between the amino acid side chains between the pairs and effecting the

248 electrostatic interacting of the cation and anion (Collins, 1997; Marcus \& Hefter, 2006; Zhou \&

249 Pang, 2017).

250 Comparing salt bridge stability in simulations and experiments

251 The free energy profiles suggest that the three salt bridges are formed and are relatively stable in

252 all but one force field, though with variations both in geometry and the depths of the different

253 minima. These observations appear to be in general conflict with the experimental NMR data

254

255

256

257

258

259

260

261

262

263

264

265

266

267

268

269 that suggest that the K12-E23 and K58-D55 pairs do not form any substantial ionic interactions, and with at most only modest interactions between the charges in K39-E35. The major exception to these general trends is the Amber ff15ipq force field where the K12-E23 and K58-D55 salt bridges are only weakly formed, and where only the K39-E35 interaction is seen at a substantial level.

As it is difficult to calculate directly the chemical shifts and isotope effects from the simulations, we opted to compare experiments and simulations indirectly by calculating the populations of the salt bridges using the free energy surfaces. In order to be conservative and not overestimate the calculated values by including e.g. solvent mediated interactions (as it is unclear how much they contribute to the experiments), we included only conformations where the nitrogen-oxygen distance is $<5.0 \AA$. The resulting populations vary between force fields and salt bridges, and range from $2 \%$ to $92 \%$, with an average value of $55 \%$ (Table 1). The salt bridge populations can also be recalculated using different definitions from the free energy profiles included as supporting data. A more detailed analysis would require a quantitative modelling of the NMR observables.

\section{Assessing convergence of simulations and effect of simulation parameters}

271

272

273

274

275

276

277

278

279

280

281

282
When MD simulations are in apparent disagreement to experiments, it is important to assess whether these differences are due to the force field and simulation parameters, or whether they can be explained e.g. by insufficient sampling. While it is difficult to prove that simulations are converged, we performed several tests that suggest that our observations are rather robust. First, as discussed above, the high degree of symmetry of the calculated free energy surfaces suggest that the individual nitrogen-oxygen distance distributions are relatively converged. Second, we calculated the free energy of salt bridge formation during the simulation (from the populations where the nitrogen-oxygen distance is $<5.0 \AA$ ) and monitored the time evolution of this (Fig. 3 shows the results from three WT-MetaD simulations with CHARMM22*/TIPS3P). While the values fluctuate for the different salt bridges, they are relatively stable in the second half of the simulations. Finally, for the K35-E39 salt bridge we also performed a parallel-tempering metadynamics simulation in CHARMM22*/TIPS3P, and compared the results to that from the 
283 WT-MetaD (Fig. 3). The two simulations converge to essentially the same free energy

284 difference. Based on these observations we estimate an error of about $0.5 \mathrm{kcal} \mathrm{mol}^{-1}$,

285 corresponding roughly to an error of 0.2 for the population of the salt bridges when the free

286 energy of salt bridge formation is close to zero.

287 Another key parameter in a simulation is the cutoff used to truncate the Lennard-Jones

288 interactions and to switch between a direct calculation of electrostatic forces and the calculations

289 of longer-range electrostatics using Ewald summation. For the K12-E23 and K35-E39 salt

290 bridges and the CHARMM22*/TIPS3P force field we therefore repeated the WT-MetaD

291 simulations varying this cutoff between $9.0 \AA-14.0 \AA$ and calculated the free energy of salt

292 bridge formation (Fig. 4). While the results vary slightly and in accordance with the estimated

293 uncertainty of our simulations, we find no systematic dependency of the free energy differences

294 on the cutoff used. Thus, these calculations suggest that the cutoff used in the simulations are

295 sufficient to obtain reasonable results.

296 Discussion

297 We have used enhance sampling simulations to analyse the stability of salt bridges in commonly

298 used fixed charge force fields for molecular dynamics simulations. We estimate the error of the

299 calculated free energies of salt bridge formation to be $\sim 0.5 \mathrm{kcal} \mathrm{mol}^{-1}$, corresponding to errors of

300 the populations of $\sim 20 \%$ (down to $10 \%$ for the most skewed populations). Surprisingly, we find

301 that the K12-E23 and K58-D55 pairs form the most stable salt bridges in most of the force fields

302 ( $64 \%$ on average across force fields) with the K39-E35 pair being distinctly less stable (on

303 average $\sim 35 \%$ ). These observations are in clear deviation from those expected from the

304 experimental observations, in that the simulations generally appear both to overestimate the

305 stability of the salt bridges, and that the order of salt bridge stability also appears to be wrongly

306 predicted. In addition to differences in the partial charges we note that differences in bonded

307 terms, e.g. in the torsional terms that affect side chain rotamers, can affect the relative stability

308 and geometry of the salt bridges.

309 The Amber ff15ipq force field is the major outlier to the above observed trends. Thus, in contrast

310 to the other force fields that overestimate the stability of the salt bridges, this force field is in

311 substantially better agreement with experiment in that it finds that the K12-E23 and K58-D55

312 salt bridges are very weakly formed ( $10 \%$ and $2 \%$, respectively). The K39-E35 salt bridge is

313 formed $\sim 20 \%$ of the time in the ff15ipq force field, and a more detailed comparison to the raw

314 NMR data would be needed to determine whether this is in agreement with experiments.

315 For the intra-helical K39-E35 interaction we note that K39 also forms transient interactions with

316 other residues (including E48 and N43), suggesting that the variations observed between force

317 fields might be the cumulative effect of a number of differences between the force fields. We

318 find that the ionic interactions are slightly stronger in TIP3PS than in standard TIP3P, in line

319 with previous observations from model compounds (Debiec, Gronenborn \& Chong, 2014). As

320 also observed before (Piana, Lindorff-Larsen \& Shaw, 2011; Debiec, Gronenborn \& Chong,

321 2014), we find that the DER correction in CHARMM22* decreases the strength of salt bridges to

322 be more in line with the experiment.

\section{Conclusions}

324 Ionic salt bridge interactions are pervasive in experimental protein structures and have in certain

325 cases been shown to contribute both to protein stability (Vaughan et al., 2002) and fast

326 association kinetics (Schreiber, Haran \& Zhou, 2009). Nevertheless, our understanding of the 
327

328

329

330

331

332

333

334

335

336

337

338

339

340

341

342

343

344

345

346

347

348

349

350

351

352

353

354

355

356

357

358

359

360

361

362

363

364

365

366

367

368

369

370

geometry and energetics of salt bridges in solution is limited by the difficulty in experimental and computational studies. While molecular dynamics simulations in explicit solvent may in principle be a quantitative and predictive model for salt bridge formation, the accuracy of such simulations hinges upon the force fields used. Building upon earlier work on model compounds (Piana, Lindorff-Larsen \& Shaw, 2011; Debiec, Gronenborn \& Chong, 2014; Debiec et al., 2016), we have here performed simulations of GB1 to determine the free energy landscape of lysine-carboxylate salt bridge formation. In these analyses we opted to examine only fixed charge force fields, but note that at least in the case of guanidinium-acetate interactions polarizable force fields have been found to give good agreement with experimental data on salt bridge strengths in model compounds (Debiec et al., 2016). Our comparison with experimental NMR data on GB1 suggest that while the force fields recapitulate the transient and weak nature of these solvent exposed ionic interactions, all but one appear to overestimate their stability slightly. This observation is in line with those from the model compounds, suggesting that together these kinds of calculations might also be used to improve the force fields. Indeed, the newly developed charge model for the Amber ff15ipq force field provides a more balanced description of salt bridge interactions for model compounds (Debiec et al., 2016), and our results on GB1 support this observation.

In general, we urge practitioners of MD simulations to take the small, but significant force field bias of many force fields into account when interpreting the importance of salt bridges observed in simulations, unless such observations are supported by experimental data. Finally, we hope that experimentalists will continue to develop approaches to study electrostatic interactions in proteins, in particular experiments that can be compared directly to simulations. Recent examples include extension of the GB1 studies to salt bridges in barnase (Williamson et al., 2013), novel NMR methods for studying electrostatics (Hass \& Mulder, 2015), NMR methods to study arginine side chains (Mackenzie \& Hansen, 2017; Yoshimura et al., 2017), and the engineering of a protein without titratable side chains as a platform for studies of protein electrostatics (Højgaard et al., 2016).

\section{Acknowledgements}

We thank the members of the Lindorff-Larsen group for advice and discussions.

\section{References}

Abascal JL., Vega C. 2005. A general purpose model for the condensed phases of water: TIP4P/2005. The Journal of chemical physics 123:234505. DOI: 10.1063/1.2121687.

Abraham MJ., Murtola T., Schulz R., Páll S., Smith JC., Hess B., Lindah E. 2015. Gromacs: High performance molecular simulations through multi-level parallelism from laptops to supercomputers. SoftwareX 1-2:19-25. DOI: 10.1016/j.softx.2015.06.001.

Banks JL., Beard HS., Cao Y., Cho AE., Damm W., Farid R., Felts AK., Halgren TA., Mainz DT., Maple JR., Murphy R., Philipp DM., Repasky MP., Zhang LY., Berne BJ., Friesner RA., Gallicchio E., Levy RM. 2005. Integrated Modeling Program, Applied Chemical Theory (IMPACT). Journal of Computational Chemistry 26:1752-1780. DOI: 10.1002/jcc.20292.

Barducci A., Bussi G., Parrinello M. 2008. Well-tempered metadynamics: A smoothly converging and tunable free-energy method. Physical Review Letters 100. DOI: 10.1103/PhysRevLett.100.020603. 
371

372

373

374

375

376

377

378

379

380

381

382

383

384

385

386

387

388

389

390

391

392

393

394

395

396

397

398

399

400

401

402

403

404

405

406

407

408

409

410

411

412

413

414

415

416

Barlow DJ., Thornton JM. 1983. Ion-pairs in proteins. Journal of Molecular Biology 168:867885. DOI: $10.1016 / \mathrm{S} 0022-2836(83) 80079-5$.

Berendsen HJC., Grigera JR., Straatsma TP. 1987. The missing term in effective pair potentials. Journal of Physical Chemistry 91:6269-6271. DOI: 10.1021/j100308a038.

Best RB., Hummer G. 2009. Optimized molecular dynamics force fields applied to the helix-coil transition of polypeptides. Journal of Physical Chemistry B 113:9004-9015. DOI: $10.1021 /$ jp901540t.

Best RB., Mittal J. 2010. Protein simulations with an optimized water model: Cooperative helix formation and temperature-induced unfolded state collapse. Journal of Physical Chemistry $B$ 114:14916-14923. DOI: 10.1021/jp108618d.

Bonomi M., Branduardi D., Bussi G., Camilloni C., Provasi D., Raiteri P., Donadio D., Marinelli F., Pietrucci F., Broglia RA., Parrinello M. 2009. PLUMED: A portable plugin for freeenergy calculations with molecular dynamics. Computer Physics Communications 180:1961-1972. DOI: 10.1016/j.cpc.2009.05.011.

Bussi G., Donadio D., Parrinello M. 2007. Canonical sampling through velocity rescaling. Journal of Chemical Physics 126. DOI: 10.1063/1.2408420.

Bussi G., Gervasio FL., Laio A., Parrinello M. 2006. Free-energy landscape for $\beta$ hairpin folding from combined parallel tempering and metadynamics. Journal of the American Chemical Society 128:13435-13441. DOI: 10.1021/ja062463w.

Casadio R., Vassura M., Tiwari S., Fariselli P., Luigi Martelli P. 2011. Correlating diseaserelated mutations to their effect on protein stability: A large-scale analysis of the human proteome. Human Mutation 32:1161-1170. DOI: 10.1002/humu.21555.

Case DA., Berryman JT., Betz RM., Cerutti DS., Cheatham III TE., Darden TA., Duke RE., Giese TJ., Gohlke H., Goetz AW., Homeyer N., Izadi S., Janowski P., Kaus J., Kovalenko A., Lee TS., LeGrand S., Li P., Luchko T., Luo R., Madej B., Merz KM., Monard G., Needham P., Nguyen H., Nguyen HT., Omelyan I., Onufriev A., Roe DR., Roitberg A., Salomon-Ferrer R., Simmerling CL., Smith W., Swails J., Walker RC., Wang J., Wolf RM., Wu X., York DM., Kollman PA. 2017. Amber 17. University of California, San Francisco. DOI: citeulike-article-id:2734527.

Collins KD. 1997. Charge density-dependent strength of hydration and biological structure. Biophysical Journal 72:65-76. DOI: 10.1016/S0006-3495(97)78647-8.

Debiec KT., Cerutti DS., Baker LR., Gronenborn AM., Case DA., Chong LT. 2016. Further along the Road Less Traveled: AMBER ff15ipq, an Original Protein Force Field Built on a Self-Consistent Physical Model. Journal of Chemical Theory and Computation 12:39263947. DOI: $10.1021 /$ acs.jctc.6b00567.

Debiec KT., Gronenborn AM., Chong LT. 2014. Evaluating the strength of salt bridges: A comparison of current biomolecular force fields. Journal of Physical Chemistry $B$ 118:6561-6569. DOI: 10.1021/jp500958r.

Dill KA. 1990. Dominant Forces in Protein Folding. Biochemistry 29:7133-7155. DOI: 10.1021/bi00483a001.

Foit L., Morgan GJ., Kern MJ., Steimer LR., von Hacht AA., Titchmarsh J., Warriner SL., Radford SE., Bardwell JCA. 2009. Optimizing Protein Stability In Vivo. Molecular Cell 36:861-871. DOI: 10.1016/j.molcel.2009.11.022.

Gallagher T., Alexander P., Bryan P., Gilliland GL. 1994. Two Crystal Structures of the B1 Immunoglobulin-Binding Domain of Streptococcal Protein $\mathrm{G}$ and Comparison with NMR. Biochemistry 33:4721-4729. DOI: 10.1021/bi00181a032. 
417 Hansmann UHE. 1997. Parallel Tempering Algorithm for Conformational Studies of Biological

$418 \quad$ Molecules. Chemical Physics Letters 281:140-150. DOI: 10.1016/S0009-2614(97)01198-6. 419 Hass MAS., Mulder FAA. 2015. Contemporary NMR Studies of Protein Electrostatics. Annual Review of Biophysics 44:53-75. DOI: 10.1146/annurev-biophys-083012-130351.

Hess B., Bekker H., Berendsen HJC., Fraaije JGEM. 1997. LINCS: A Linear Constraint Solver for molecular simulations. Journal of Computational Chemistry 18:1463-1472. DOI: 10.1002/(SICI)1096-987X(199709)18:12<1463::AID-JCC4>3.0.CO;2-H.

Højgaard C., Kofoed C., Espersen R., Johansson KE., Villa M., Willemoës M., Lindorff-Larsen K., Teilum K., Winther JR. 2016. A Soluble, Folded Protein without Charged Amino Acid Residues. Biochemistry 55:3949-3956. DOI: 10.1021/acs.biochem.6b00269.

Hornak V., Abel R., Okur A., Strockbine B., Roitberg A., Simmerling C. 2006. Comparison of multiple amber force fields and development of improved protein backbone parameters. Proteins: Structure, Function and Genetics 65:712-725. DOI: 10.1002/prot.21123.

Jacobsen CF., Linderstrøm-Lang K. 1949. Salt linkages in proteins. Nature 164:411-412. DOI: $10.1038 / 164411 \mathrm{a} 0$.

Jensen KP. 2008. Improved Interaction Potentials for Charged Residues in Proteins. The Journal of Physical Chemistry B 112:1820-1827. DOI: 10.1021/jp077700b.

Jensen M., Jogini V., Borhani DW., Leffler AE., Dror RO., Shaw DE. 2012. Mechanism of voltage gating in potassium channels. Science 336:229-233. DOI:

$10.1126 /$ science. 1216533.

Jorgensen WL., Chandrasekhar J., Madura JD., Impey RW., Klein ML. 1983. Comparison of simple potential functions for simulating liquid water. The Journal of Chemical Physics 79:926-935. DOI: 10.1063/1.445869.

Kumar S., Nussinov R. 2002. Close-range electrostatic interactions in proteins. ChemBioChem 3:604-617. DOI: 10.1002/1439-7633(20020703)3:7<604::AID-CBIC604>3.0.CO;2-X.

Linderstrøm-Lang K. 1924. On the ionization of proteins. CR Trav. Lab. Carlsberg 15:70-95. Lindorff-Larsen K., Piana S., Palmo K., Maragakis P., Klepeis JL., Dror RO., Shaw DE. 2010. Improved side-chain torsion potentials for the Amber ff99SB protein force field. Proteins: Structure, Function and Bioinformatics 78:1950-1958. DOI: 10.1002/prot.22711.

Mackenzie HW., Hansen DF. 2017. A13C-detected15N double-quantum NMR experiment to probe arginine side-chain guanidinium15Nnchemical shifts. Journal of Biomolecular NMR 69:123-132. DOI: 10.1007/s10858-017-0137-2.

MacKerell AD., Bashford D., Bellott M., Dunbrack RL., Evanseck JD., Field MJ., Fischer S., Gao J., Guo H., Ha S., Joseph-McCarthy D., Kuchnir L., Kuczera K., Lau FTK., Mattos C., Michnick S., Ngo T., Nguyen DT., Prodhom B., Reiher WE., Roux B., Schlenkrich M., Smith JC., Stote R., Straub J., Watanabe M., Wiórkiewicz-Kuczera J., Yin D., Karplus M. 1998. All-Atom Empirical Potential for Molecular Modeling and Dynamics Studies of Proteins. The Journal of Physical Chemistry B 102:3586-3616. DOI: 10.1021/jp973084f.

Mackerell AD., Feig M., Brooks CL. 2004. Extending the treatment of backbone energetics in protein force fields: Limitations of gas-phase quantum mechanics in reproducing protein conformational distributions in molecular dynamics simulation. Journal of Computational Chemistry 25:1400-1415. DOI: 10.1002/jcc.20065.

Marcus Y., Hefter G. 2006. Ion pairing. Chemical Reviews 106:4585-4621. DOI: $10.1021 / \mathrm{cr} 040087 \mathrm{x}$.

Nielsen S V., Stein A., Dinitzen AB., Papaleo E., Tatham MH., Poulsen EG., Kassem MM., Rasmussen LJ., Lindorff-Larsen K., Hartmann-Petersen R. 2017. Predicting the impact of 
463

464

465

466

467

468

469

470

471

472

473

474

475

476

477

478

479

480

481

482

483

484

485

486

487

488

489

490

491

492

493

494

495

496

497

498

499

500

501

502

503

504

505

506

507

508

Lynch syndrome-causing missense mutations from structural calculations. PLoS Genetics 13. DOI: 10.1371/journal.pgen.1006739.

Parrinello M., Rahman A. 1981. Polymorphic transitions in single crystals: A new molecular dynamics method. Journal of Applied Physics 52:7182-7190. DOI: 10.1063/1.328693.

Parrinello M., Rahman A. 1982. Strain fluctuations and elastic constants. The Journal of Chemical Physics 76:2662-2666. DOI: 10.1063/1.443248.

Piana S., Donchev AG., Robustelli P., Shaw DE. 2015. Water dispersion interactions strongly influence simulated structural properties of disordered protein states. Journal of Physical Chemistry B 119:5113-5123. DOI: 10.1021/jp508971m.

Piana S., Lindorff-Larsen K., Shaw DE. 2011. How robust are protein folding simulations with respect to force field parameterization? Biophysical Journal 100. DOI: 10.1016/j.bpj.2011.03.051.

Robustelli P., Piana S., Shaw DE. 2018. Developing a molecular dynamics force field for both folded and disordered protein states. PNAS 0260. DOI: 10.1073/pnas.1800690115.

Sarakatsannis JN., Duan Y. 2005. Statistical characterization of salt bridges in proteins. Proteins: Structure, Function and Genetics 60:732-739. DOI: 10.1002/prot.20549.

Schreiber G., Haran G., Zhou HX. 2009. Fundamental aspects of protein - Protein association kinetics. Chemical Reviews 109:839-860. DOI: 10.1021/cr800373w.

Schueler O., Margalit H. 1995. Conservation of salt bridges in protein families. Journal of Molecular Biology 248:125-135. DOI: 10.1006/jmbi.1995.0206.

Serrano L., Horovitz A., Avron B., Bycroft M., Fersht AR. 1990. Estimating the Contribution of Engineered Surface Electrostatic Interactions to Protein Stability by Using Double-Mutant Cycles. Biochemistry 29:9343-9352. DOI: 10.1021/bi00492a006.

Speakman, J. B., \& Hirst MC. 1931. Constitution of the keratin molecule. Nature 128:1073. van der Spoel D., Lindahl E., Hess B., van Buuren a. R., Apol E., Meulenhoff PJ., Tieleman DP., Sijbers a. LTM., Feenstra K a., van Drunen R., Berendsed HJC. 2010. GROMACS User Manual version 4.5.6. Www.Gromacs.Org:7778. DOI: 10.1007/SpringerReference_28001.

Steinbrecher T., Zhu C., Wang L., Abel R., Negron C., Pearlman D., Feyfant E., Duan J., Sherman W. 2017. Predicting the Effect of Amino Acid Single-Point Mutations on Protein Stability - Large-Scale Validation of MD-Based Relative Free Energy Calculations. Journal of Molecular Biology 429:948-963. DOI: 10.1016/j.jmb.2016.12.007.

Sutto L., Gervasio FL. 2013. Effects of oncogenic mutations on the conformational free-energy landscape of EGFR kinase. Proceedings of the National Academy of Sciences 110:1061610621. DOI: 10.1073/pnas. 1221953110.

Takano K., Tsuchimori K., Yamagata Y., Yutani K. 2000. Contribution of salt bridges near the surface of a protein to the conformational stability. Biochemistry 39:12375-12381. DOI: 10.1021/bi000849s.

Tomlinson JH., Ullah S., Hansen PE., Williamson MP. 2009. Characterization of salt bridges to lysines in the protein G B1 domain. Journal of the American Chemical Society 131:46744684. DOI: $10.1021 /$ ja808223p.

Tribello GA., Bonomi M., Branduardi D., Camilloni C., Bussi G. 2014. PLUMED 2: New feathers for an old bird. Computer Physics Communications 185:604-613. DOI: 10.1016/j.cpc.2013.09.018.

Vaughan CK., Harryson P., Buckle AM., Fersht AR. 2002. A structural double-mutant cycle: Estimating the strength of a buried salt bridge in barnase. Acta Crystallographica Section 
509 D: Biological Crystallography 58:591-600. DOI: 10.1107/S0907444902001567.

510 Williamson MP., Hounslow AM., Ford J., Fowler K., Hebditch M., Hansen PE. 2013. Detection

511 of salt bridges to lysines in solution in barnase. Chem. Commun. 49:9824-9826. DOI:

$512 \quad 10.1039 /$ C3CC45602A.

513 Yoshimura Y., Oktaviani NA., Yonezawa K., Kamikubo H., Mulder FAA. 2017. Unambiguous

514 Determination of Protein Arginine Ionization States in Solution by NMR Spectroscopy.

515 Angewandte Chemie - International Edition 56:239-242. DOI: 10.1002/anie.201609605.

516 Zhou H., Pang X. 2017. Electrostatic Interactions in Protein Structure, Folding,Binding, and Condensation. Chemical Reviews. DOI: 10.1021/acs.chemrev.7b00305. 


\section{Table $\mathbf{1}$ (on next page)}

Simulations, force fields and salt bridge stabiliy.

The table shows the stability of each of the three salt bridges, reported as the population of structures with $\mathrm{N}-\mathrm{O}$ distances shorter than $5 \AA$, in each of the eight different combinations of force field and water models. 


\section{Table 1}

\begin{tabular}{|c|c|c|c|c|c|}
\hline \multirow{2}{*}{ Force field } & \multirow{2}{*}{ Water model } & $\boldsymbol{d}_{\boldsymbol{m a x}}$ & \multicolumn{4}{|c|}{ Population } \\
\cline { 4 - 6 } & & & $\begin{array}{c}\text { K12- } \\
\text { E23 }\end{array}$ & $\begin{array}{c}\text { K39- } \\
\text { D35 }\end{array}$ & $\begin{array}{c}\text { K58- } \\
\text { D55 }\end{array}$ \\
\hline CHARMM22* & TIPS3P & $13.5 \AA$ & 0.73 & 0.42 & 0.61 \\
\hline CHARMM22* & TIP3P & $13.5 \AA$ & 0.67 & 0.20 & 0.61 \\
\hline CHARMM27 & TIPS3P & $13.5 \AA$ & 0.83 & 0.14 & 0.75 \\
\hline Amber ff03w & TIP4P/2005 & $13.5 \AA$ & 0.60 & 0.48 & 0.81 \\
\hline Amber ff99SB*-ILDN & TIP3P & $13.5 \AA$ & 0.63 & 0.72 & 0.98 \\
\hline OPLS AA & SPC/E & $13.5 \AA$ & 0.65 & 0.39 & 0.98 \\
\hline a99SB-disp & TIP4P-D & $13.0 \AA$ & 0.67 & 0.25 & 0.68 \\
\hline AMBER ff15ipq & SPC/E & $14.0 \AA$ & 0.096 & 0.20 & 0.02 \\
\hline
\end{tabular}




\section{Figure 1}

Formation and stability of salt bridges in the B1 domain of Protein G (GB1).

In panel A we show the structure of GB1 in cartoon representaion, highlighting the location of the three salt bridges that are found in crystal structures of GB1. In B-D we show free energy profiles of salt bridge formation as obtained from metadynamics simulations with the CHARMM 22* force field and TIPS3P water model. The different inserts illustrate representative structures and show both contact ion pairs where the two residues are in direct interactions, and solvent-separated ion pairs where one or more water molecules sit between the charged residues.

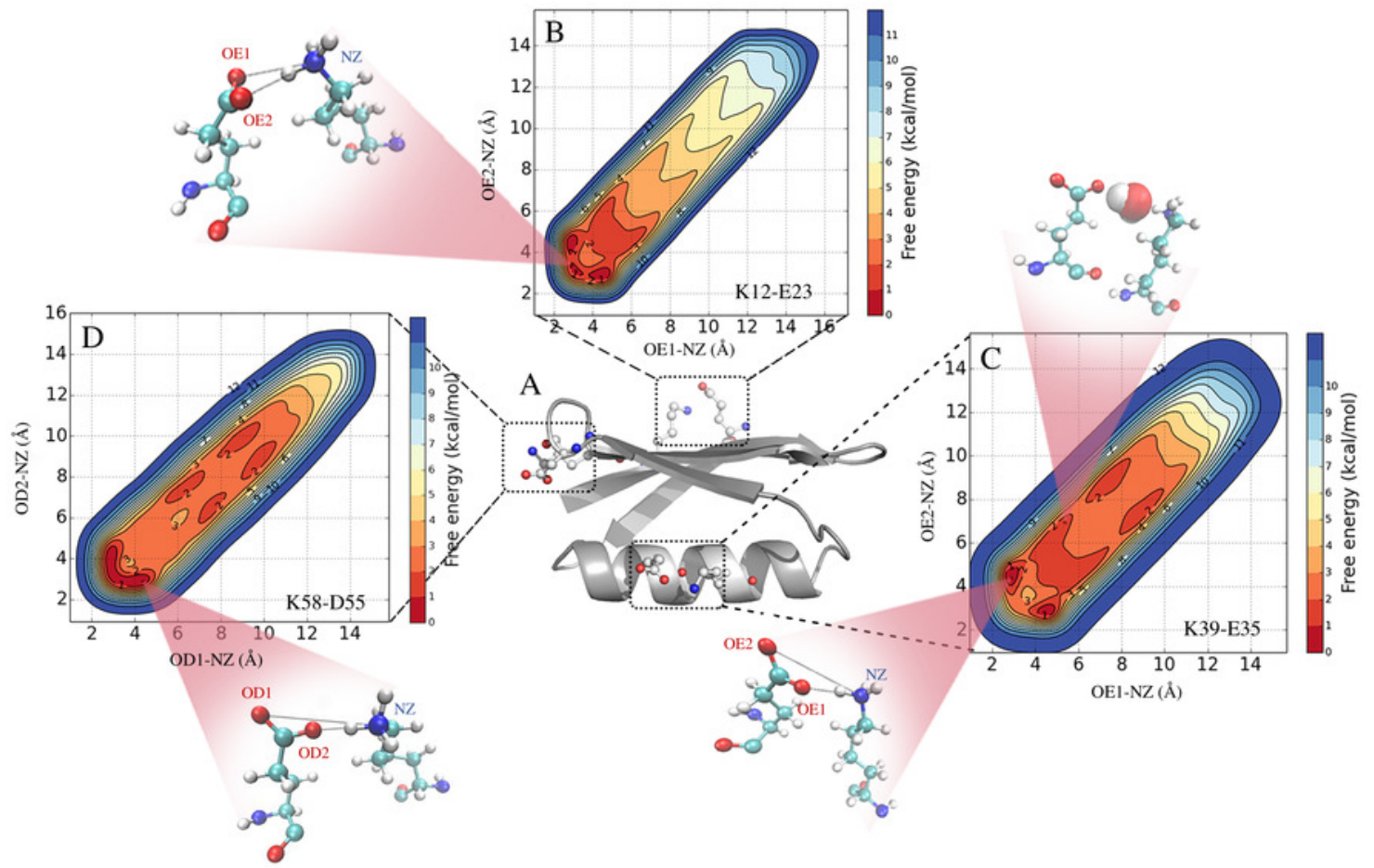




\section{Figure 2}

Salt bridge formation using both unbiased and enhanced sampling molecular dynamics simulations.

Using the K12-E23 salt-bridge and the CHARMM22*TIPS3P force field as an example, we compare (A) unbiased simulations with (B) metadynamics simulations. In panel B we also show how the restraint energy acts to avoid that the ion pairs to form excessively long distances. Note that since the metadynamics simulation is biased, the resulting distribution of distances is not expected to be the same until after this bias has been removed. This unbiasing was performed before calculating the free energy profiles in all other figures and analyses. 

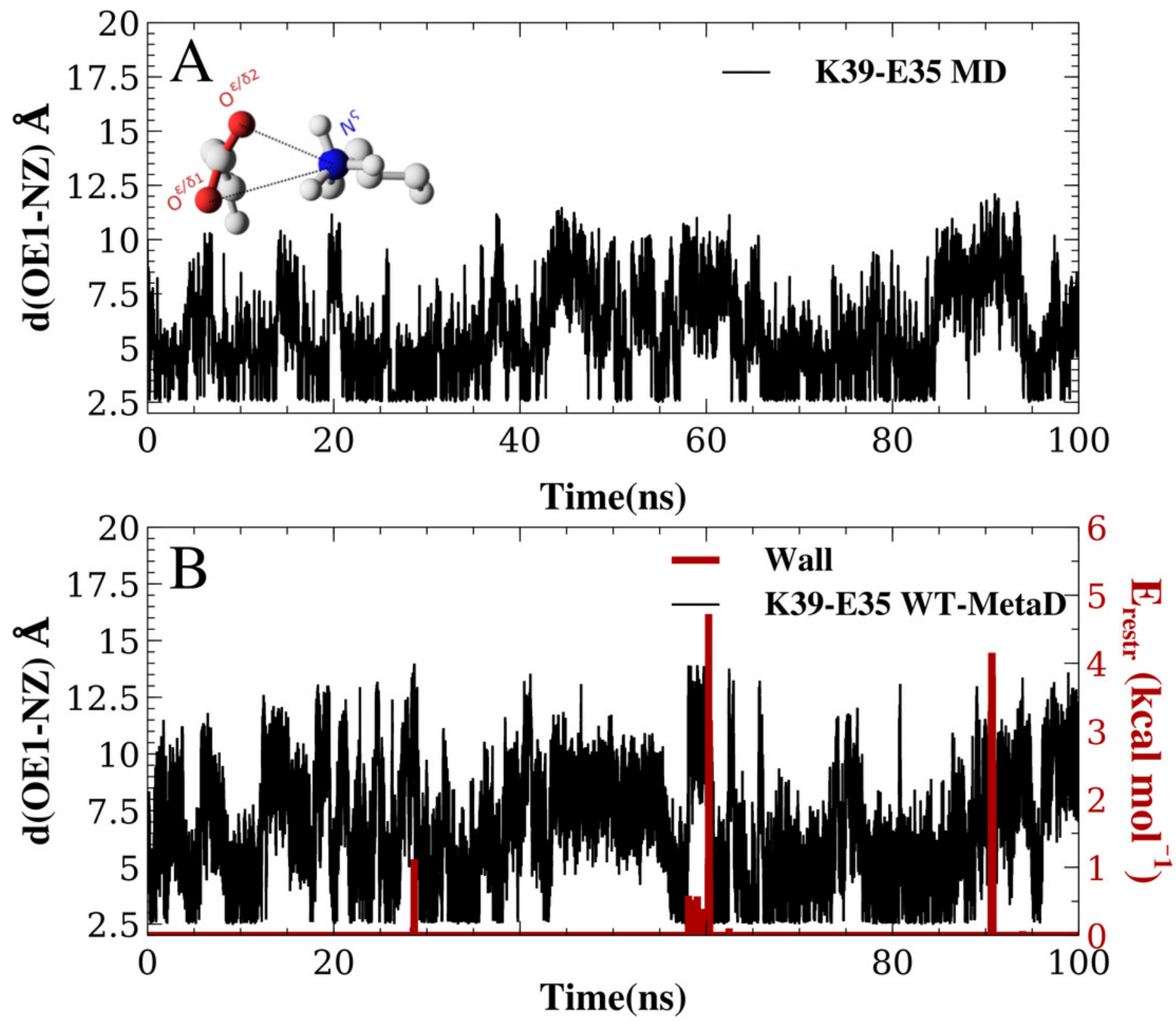


\section{Figure 3}

Assessing the convergence of the free energy differences.

In the figure we show how the free energy of salt bridge formation as a function of time, using the CHARMM22*/TIPS3P force field as an example. Initially, the values for the three different salt bridges fluctuate, but eventually converge after $100 \mathrm{~ns}$ of simulation (solid lines, bottom axis). We also performed a PT-MetaD simulation for the of K39-E35 salt bridge as an alternative approach to determine the free energy landscape (dashed line). After initial fluctuations, the free energy difference converges after $50 \mathrm{~ns}$ (top axis) to a value close to that obtained using WT-MetaD. 


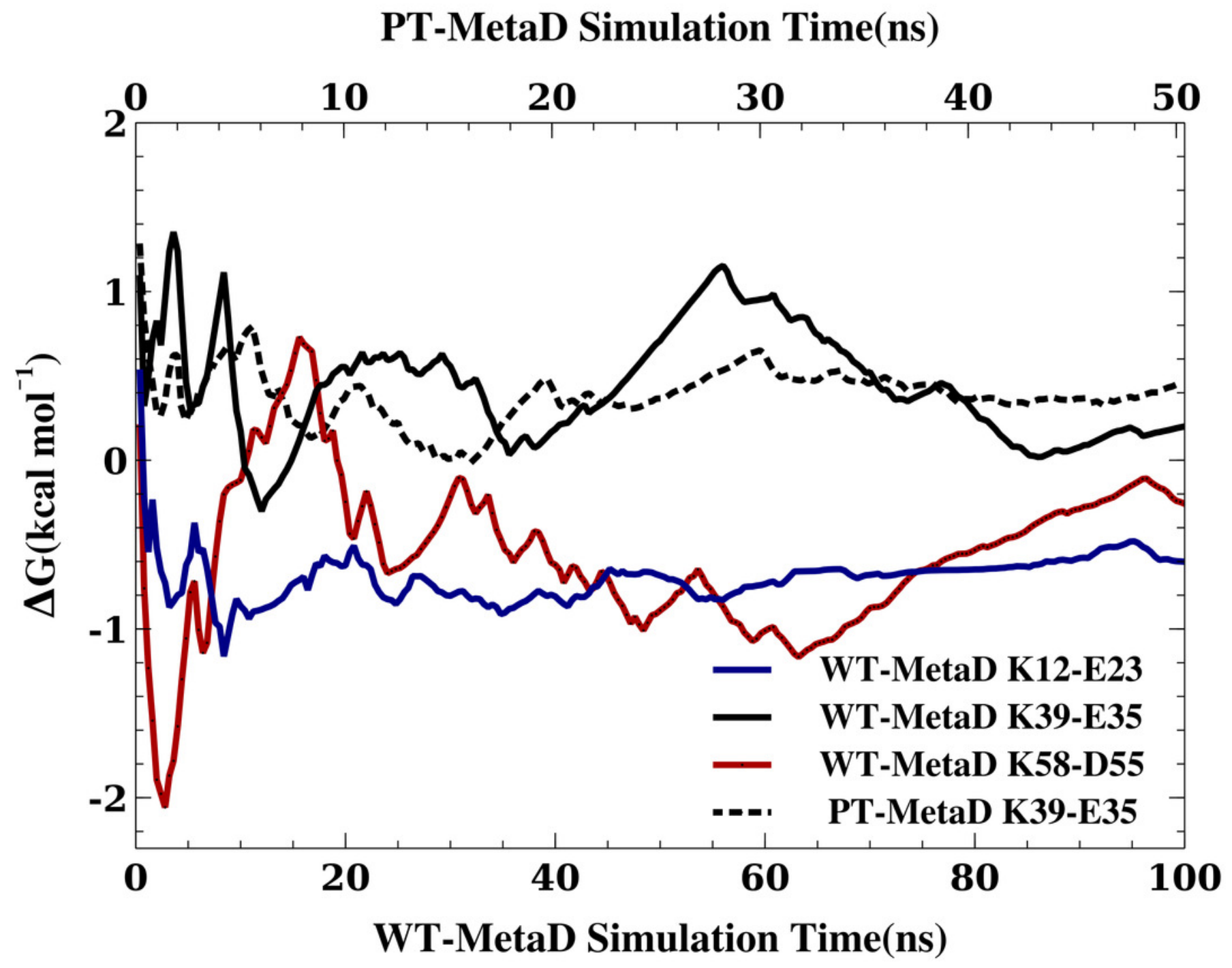


Figure 4

Assessing the effect of varying the cutoff distance.

We repeated the WT-MetaD for two of the salt bridges (K12-E23 and K39-E35) but varying the cutoff for the Lennard-Jones interactions and for switching from the direct calculation of electrostatic interactions to Ewald summation. The figure shows the effect of varying this cutoff on the stability of these two salt bridges.

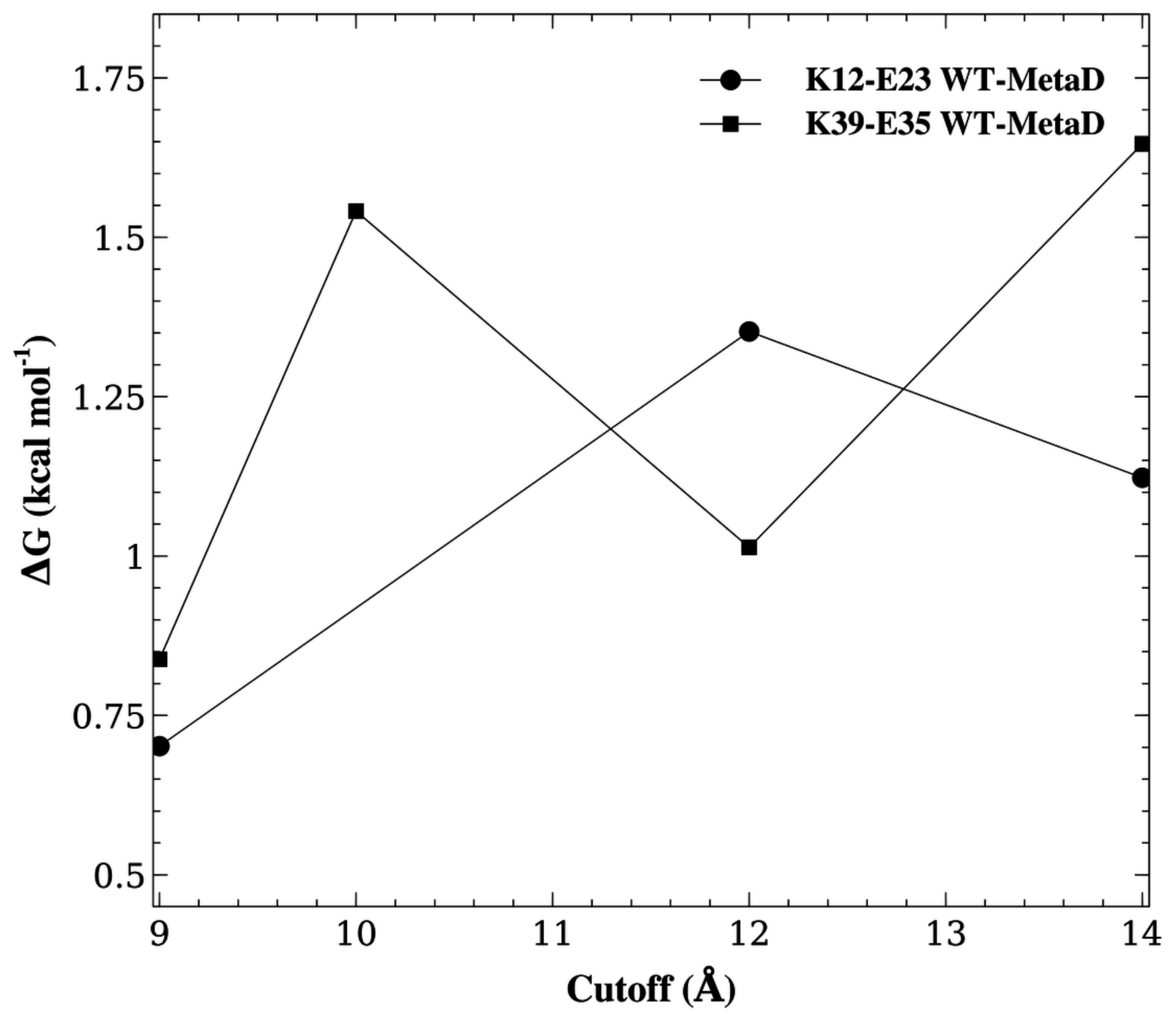

